\title{
Clinico-etiological study of vaginal discharge in adult women - A hospital based study
}

\author{
Vani Gopal ${ }^{1}$, Gopal R. ${ }^{2}$, Rupavani ${ }^{3}$, Mangaiyarkarasi T.,"* \\ ${ }^{1}$ Senior Medical Officer, Dept. of Microbiology, Rajiv Gandhi Government Women \& Children Hospital, Puducherry, ${ }^{2}$ Professor \\ and Head, ${ }^{3}$ Assistant Professor, ${ }^{4}$ Associate Professor, ${ }^{2,4}$ Dept. of Microbiology, ${ }^{3}$ Dept. of Obstetrics \& Gynaecology, ${ }^{2,4}$ Sri \\ Manakula Vinayagar Medical College and Hospital, Puducherry, ${ }^{3}$ Indira Gandhi Medical College and Research Institute, \\ Puducherry, India
}

\section{*Corresponding Author: Mangaiyarkarasi T}

Email: drtmangai76@yahoo.in

\begin{abstract}
Introduction: The common complaint in women of reproductive age group is discharge per vaginum. The etiology may be due to physiological or pathological. Infectious causes include Bacterial vaginosis, candidiasis and trichomoniasis. Precise diagnosis of the infectious cause helps in initiation of appropriate treatment avoiding misuse of drugs as in the syndromic approach.

Objective: To find the aetiopathogenesis for vaginal discharge and their prevalence in symptomatic adult women attending the outpatient department of Obstetrics and Gynaecology of our hospital during the study period.

Materials and Methods: A cross - sectional hospital based study carried out from November 2012 to January 2014. Swabs were collected from all symptomatic adult women aged 15-45 years and examined by wet mount, Gram stain and inoculated on Sabourauds Dextrose Agar (SDA) medium for isolation of Candida species.

Results: The prevalence rate of bacterial vaginosis, vaginal candidiasis and trichomoniasis were $32 \%, 25 \%$ \& $8.5 \%$ respectively. Conclusion: The most common etiology for vaginal discharge in our study was bacterial vaginosis.
\end{abstract}

Keywords: Vaginitis, Bacterial vaginosis, Trichomoniasis, Candidiasis, Syndromic approach.

\section{Introduction}

Discharge per vaginum is the most common complaint among women of reproductive age group. ${ }^{1}$ $90 \%$ of infectious causes of vaginal discharge include bacterial vaginosis followed by candidiasis and trichomoniasis. Other infectious causes include Chlamydiatrachomatis and Neisseria gonorrhoeae. ${ }^{2}$ The common causes of physiological discharge are use of contraceptives, menstruation and pregnancy. ${ }^{3}$ The associated symptoms produced by pathological discharge include itching, burning sensation, offensive odor and dyspareunia. The management of vaginal discharge is often based on syndromic approach. The main disadvantage of this approach is misdiagnosis and injudicious treatment with multiple antimicrobials leading to development of drug resistant strains and economic burden on the patient. Simple laboratory tests like gram staining, wet mount, whiff test etc can help in establishing the etiological agent and institution of the appropriate therapy. The present study aims to identify the etiology for vaginal discharge based on simple laboratory tests and establish the utility of these tests in management.

\section{Materials and Methods}

After the approval from Institutional Ethics Committee across sectional hospital based study was carried out over a period of 2 years (November 2012 January 2014). All women of age group 18 to 45 years who attended gynaecology OPD of our hospital presenting with complaints of vaginal discharge were included in the study after obtaining an informed consent. A validated proforma was used for recording the complaints, personal history, menstrual history, coexisting diabetes mellitus, use of drugs including oral contraceptive pills, antibiotics, antifungals, antiprotozoals in the recent past.

\section{Inclusion Criteria}

1. Non pregnant women in the age group of $18-45$ years with complaints of discharge per vagina.

\section{Exclusion Criteria}

1. Menstruating women

2. Women in the post-abortal period

3. Women in the post-operative period

4. Post menopausal women

5. Women with intra-uterine contraceptive device

A total of 200 consecutive patients attending the OPD during the study period with the complaints of vaginal discharge were included in the study as per the above criteria.

A vaginal examination was done using a sterile unlubricated Cusco's speculum while noting the character of the discharge i.e. color, odor, amount and presence of any erosions over the cervical os. Three high vaginal swabs were collected from the upper part of the posterior fornix and the first swab was put in a test tube containing $0.2 \mathrm{ml}$ of $5 \%$ glucose saline which was subjected to wet mount examination within 15 minutes of collection. The second swab was used for determining the $\mathrm{pH}$, whiff test and Gram stain while the third swab was used for inoculation on SDA with antibiotics. The plates were incubated at $37^{\circ} \mathrm{C}$ for two weeks. 
The wet mount preparation was examined under $40 \mathrm{X}$ objective for the presence of Trichomonasvaginalis (Fig. 1) and also for the presence of clue cells and yeast cells. The gram stained smear was examined for the presence of yeast cells, clue cells suggestive of Bacterial vaginosis and gonococci (Fig. 2). Nugent's scoring was used for diagnosis of bacterial vaginosis. Cultures were examined every other day for growth of yeasts for two weeks. Any yeast grown were identified by Gram stain, germ tube test, chlamydospore production, sugar fermentation, pigment production on chrom agar. All media used were procured from Himedia Pvt. Ltd.

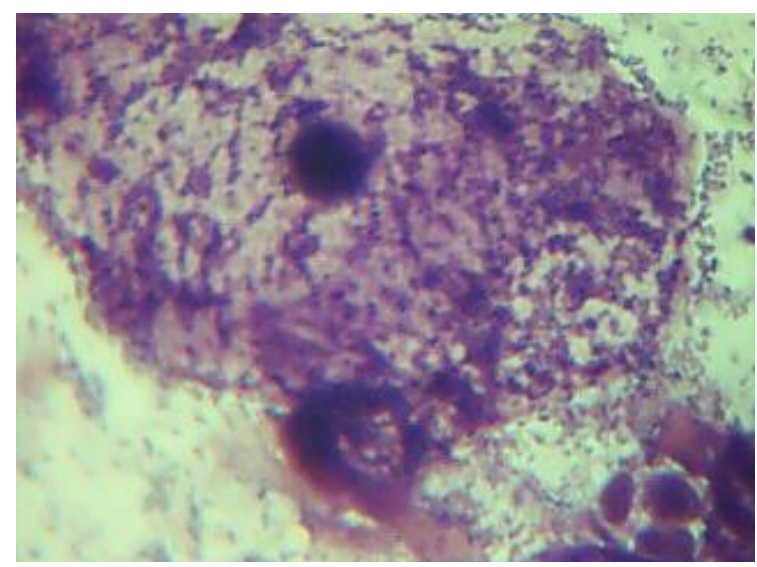

Fig. 1: Gram stain showing clue cells

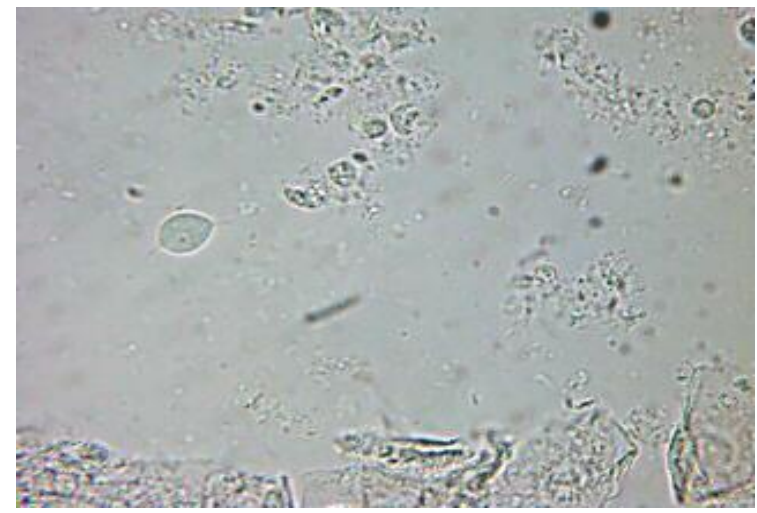

Fig. 2: Wet mount of Trichomonasvaginalis

\section{Results}

The age wise distribution of the study population is shown in (Table 1). About $30.5 \%$ of study population belongs to age group of 26-30 years. The most common etiology for vaginal discharge in our study was bacterial vaginosis $(32 \%)$ and was more common among multiparous women. Vaginal candidiasis was found in $50(25 \%)$ whereas Trichomoniasis wasfoundin17outof 200 patients (Table 2). The predominant species of Candida isolated was Candida albicans (Table 3).
Table 1: Age- wise distribution of study population $(\mathrm{n}=\mathbf{2 0 0})$

\begin{tabular}{|l|c|}
\hline Age (Years) & Number $(\%)$ \\
\hline $20-25$ & $25(12.5)$ \\
\hline $26-30$ & $61(30.5)$ \\
\hline $31-35$ & $47(23.5)$ \\
\hline $36-40$ & $53(26.5)$ \\
\hline $40-42$ & $14(7)$ \\
\hline Total & $200(100)$ \\
\hline
\end{tabular}

Table 2: Distribution of different etiological agents $(\mathbf{n}=\mathbf{2 0 0})$

\begin{tabular}{|l|c|}
\hline Etiological agent & Numbers (\%) \\
\hline Bacterial vaginosis & $64(32)$ \\
\hline Candidiasis & $50(25)$ \\
\hline Tricomoniasis & $17(8.5)$ \\
\hline Mixed infection & $8(4)$ \\
\hline
\end{tabular}

Table 3: Candida species isolated $(n=50)$

\begin{tabular}{|l|c|}
\hline \multicolumn{1}{|c|}{ Candida species } & Number of isolates \\
\hline C. albicans & $25(50 \%)$ \\
\hline C. tropicalis & $9(18 \%)$ \\
\hline C. parapsilosis & $10(20 \%)$ \\
\hline C. krusei & $2(4 \%)$ \\
\hline C. glabrata & $4(8 \%)$ \\
\hline
\end{tabular}

\section{Discussion}

Discharge per vaginum is a common complaint among women in the reproductive age group. The discharge may be physiological or pathological. The pathogens associated with vaginal discharge include bacteria, fungi, viruses and protozoan parasites. The common bacterial agents include Neisseria gonorrhoeae, Gardnerella vaginalis, fungi include Candida species and the protozoa Trichomonas vaginalis and Herpes simplex virus. ${ }^{4}$ The percentage of etiological agents varies with geographical location, education, personal hygiene, economical status. Bacterial vaginosis accounts for approximately onethird of vulvo-vaginal infections. It is a polymicrobial syndrome and the causative agents include Gardnerella vaginalis, Mobilincus, Bacteroides and Mycoplasma hominis. ${ }^{5}$ It is characterized by profuse, malodorous discharge and associated with severe adverse outcomes like preterm birth, pelvic inflammatory disease and high risk of acquiring HIV infection. ${ }^{6}$

Vaginal candidiasis, which is characterized by curd like discharge and pruritisis, commonly associated with diabetes, pregnancy and prolonged use of antibiotics. ${ }^{7}$ Non albicans Candida is being more often isolated from these cases in the recent times which are often resistant to azole group of drugs. ${ }^{7-9}$

Patients with trichomoniasis, a sexually transmitted disease present with copious yellow or green frothy discharge. ${ }^{9}$

In the present study microbiological diagnosis was made in $69.5 \%$ of the cases and about $43 \%$ were of 20 
to 30 years age group. All the women participated in the study were married and $93.5 \%$ of them were multiparous. In the present study prevalence of bacterial vaginosis $(32 \%)$ was highest which is similar to the study by Bhalla et al. ${ }^{10}$ Whereas a much higher rate (45\%) was found in the study by Puri et $\mathrm{al}^{11}$ and a lower rate of $24 \%$ in the study by Rekha et al Bangalore, ${ }^{12}$ $23 \%$ in study by Pavani et al. ${ }^{13}$ The variation in the rates may be due to varied geographical distribution, differences in the socio-economic status and method used in the diagnosis. In the present study presence of clue cells had the best correlation with the Nugent's scoring. Whiff test was found to give a large number of false negative results. Only $37.5 \%$ bacterial vaginosis was clinically diagnosed others had been missed or wrongly diagnosed. In our study the prevalence of Trichomonas vaginalis was $8.5 \%$ and Trichomonas vaginalis was predominant in the age group of 36-42 years. Studies have shown that Trichomonas vaginalis infection increases with age. ${ }^{14}$ The most common associated symptoms were burning sensation in the vagina, dyspareunia, dysuria and pruritis. A similar rate of prevalence was observed in the study by Madhivanan et al. ${ }^{15}$ The prevalence rate of Trichomonas vaginalis in India ranges from 6 to $10 \% .^{16}$ Vulvo-vaginal candidiasis was the second most common cause $(25 \%)$ of vaginal discharge and Candida albicans was the predominant species isolated in our study. The common associated symptom was curdy white discharge (80\%), pruritis $(76 \%)$ followed by dysuria, burning sensation in the vagina, dyspareunia which were similar to a study by Aruna Verma et al (2013). ${ }^{17}$ In our study only $66 \%$ of microbiologically diagnosed cases were diagnosed clinically and $34 \%$ were undiagnosed and about $8 \%$ of cases of candidiasis were over-treated with antifungal drugs. This highlights the need for laboratory diagnosis of vaginal specimens before commencing therapy.

\section{Conclusion}

In our study also bacterial vaginosis is the most common cause of symptomatic vaginal discharge followed by candidiasis and trichomoniasis. Simple microscopic examination like wet mount and Gram stain are useful tools for rapid and precise diagnosis which helps in institution of appropriate therapy thus reducing the costs, failure of therapy, injudicious use of antimicrobials and prolonged suffering and unfavorable outcome.

\section{References}

1. Jabeen N, Soomro U. Bacterial vaginosis. Gynaecologist, 2001;5:56-7.

2. Sahoo B, Bhandri H, Shavma M, Malhotra S, Sawhney $\mathrm{H}$, Komar B. Role of male partner in lower genitourinary tract. Indian J Med Res. 2000;112:90-94.

3. Vermeulen GM, Van ZAA, Bruinse HW. Changes in vaginal flora after $2 \%$ clindamycin vaginal cream in women at high risk of spontaneous preterm birth. BJOG. 2001;108:697-700.
4. Hoosen AA. Management of vaginal discharge, CME February 2004 vol22 no2.

5. Krohn M, Hillier S, Eschenbach D. comparison of methods for diagnosing bacterial vaginosis among pregnant women. J Clin Microbiol.1989;27:1266-71.

6. Hillier SL, Nugent RP, Eschenbach DA. association between bacterial vaginosis and preterm delivery of a low-birth-weight infant- The Vaginal Infections and Prematurity Study Group. NEJM. 1995;333(26):1737-42.

7. Agarwal S, Manchanda V, Verma N, Bhalla P. Yeast identification in routine clinical microbiology laboratory and its clinical relevance. Indian J Med Microbiol. 2011;29:172-7.

8. Robertson W. Mycology of vulvo-vaginitis. Am J Obstet Gynecol. 1988; 158:989-93.

9. Vijaya D, Harsha TR, Nagarathnamma T. Candida speciation using chrom agar. J Clin Diagn Res. 2011;5:755-7.

10. Bhalla P, Chawla R, Garg S, Singh MM, Raina U, Bhalla $\mathrm{R}$, et al. prevalence of bacterial vaginosis among women in Delhi, India. Indian J Med Res. 2007;125(2):167-72.

11. Puri KJ, Madan A, Bajaj K. incidence of various causes of vaginal discharge among sexually active females in age group 20-40 years. Indian Journal of Dermatology, Venereology and Leprology. 2003;69:122-25.

12. Rekha S, Jyothi S. comparison of visual, clinical and microbiological diagnosis of symptomatic vaginal discharge in the reproductive age group. Int J Pharm Biomed Res. 2010;1(4):144-48.

13. Pavani K, Saileela K. diagnosis of bacterial vaginosis in reproductive age group in tertiary health care hospital in south India. Journal of Evolution of Medical and Dental Sciences. 2013;12:6611-15.

14. Johnston VJ, Mabey DC. Global epidemiology and control of Trichomonas vaginalis. Curr Opin Infect Dis. 2008;21:56-64.

15. Madhivanan P, Bartman MT, Pasuti L. prevalence of Trichomonas vaginalis infection among young reproductive age women in India:implications for treatment and prevention. Sex Health. 2009;6:339-44.

16. Kaur S, Khurana S, Bagga R, Wanchu A, Malla N. trichomoniasis among women in north India: A hospital based study. Indian Sex Trans Dis and AIDS. 2008; 29:276-81.

17. Aruna Verma, Abhilasha Gupta, Swati Goel, Amit Garg. Clinicopathological correlation of infective vaginal discharges in non pregnant sexually active women of reproductive age group in a tertiary care centre of Western U.P. Int J Reprod Contracept Obstet Gynecol. 2013;2(3):349-54.

How to cite this article: Gopal V, Gopal R, Rupavani, Mangaiyarkarasi T. Clinico-etiological study of vaginal discharge in adult women - A hospital based study. Indian J Microbiol Res. 2018;5(4):535-537. 